\title{
Fast Tiered Labeling with Topological Priors
}

\author{
Ying Zheng, Steve Gu, and Carlo Tomasi \\ Duke University, U.S.A. \\ \{yuanqi, steve, tomasi\}@cs.duke.edu
}

\begin{abstract}
We consider labeling an image with multiple tiers. Tiers, one on top of another, enforce a strict vertical order among objects (e.g. sky is above the ground). Two new ideas are explored: First, under a simplification of the general tiered labeling framework proposed by Felzenszwalb and Veksler [1], we design an efficient $O(K N)$ algorithm for the approximate optimal labeling of an image of $N$ pixels with $K$ tiers. Our algorithm runs in over 100 frames per second on images of VGA resolutions when $K$ is less than 6 . When $K=3$, our solution overlaps with the globally optimal one by Felzenszwalb and Veksler in over $99 \%$ of all pixels but runs 1000 times faster. Second, we define a topological prior that specifies the number of local extrema in the tier boundaries, and give an $O(N M)$ algorithm to find a single, optimal tier boundary with exactly $M$ local maxima and minima. These two extensions enrich the general tiered labeling framework and enable fast computation. The proposed topological prior further improves the accuracy in labeling details.
\end{abstract}

\section{Introduction}

We consider labeling an image with multiple tiers. Tiers, one on top of another, enforce a strict vertical order among objects. For example, the sky is above the ground and bottles are placed on top of a table. For indoor images, the ceiling is above the wall above the floor. In general, ordering may come from physical laws like gravity or typical object arrangements, and is commonly seen in daily life pictures. Figure 1 illustrates the setting for tiered labeling.

Other than the strict order among objects, we often have certain prior knowledge that is useful for object labeling. One important prior is the regularity of the shape of an object. A commonly used measure is the total variation of a boundary curve, which only guarantees that a curve is locally smooth. We define instead the topological smoothness, which bounds and specifies the number of local extrema of a shape boundary, and is useful for enforcing that a curve is globally smooth and has a given number of peaks or valleys (Figure 2). We explore this novel, topological prior in the multi-tier labeling framework.

\subsection{Literature Review}

Scene labeling assigns to each pixel a semantic label and has been widely studied $[2-7,1,8]$. Let $f: \Omega \rightarrow \mathcal{L}$ be a labeling function mapping from the image grid 

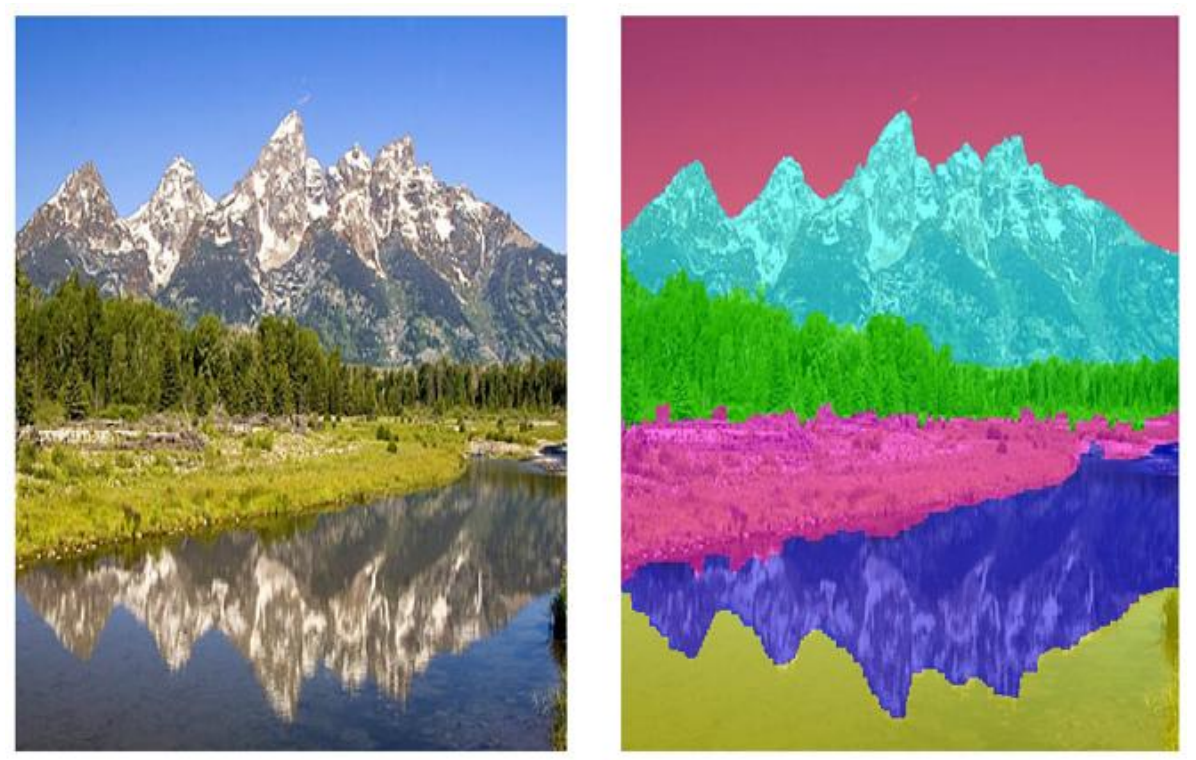

Fig. 1. The tiered labeling problem partitions an input image (left) into multiple tiers (right). Each tier (6 in total) is displayed with a different color. The labeling takes less than 0.01 seconds on an image of resolution $640 \times 480$.

$\Omega$ to the label space $\mathcal{L}$. Let $f_{p}$ be the label of pixel $p$. Labeling can often be modeled as minimizing an energy function in the form of a Markov Random Field (MRF)[2]:

$$
\min _{f}\{\sum_{p \in \Omega} \underbrace{D_{p}\left(f_{p}\right)}_{\text {data cost }}+\lambda \sum_{(p, q) \in \mathcal{N}} \underbrace{V\left(f_{p}, f_{q}\right)}_{\text {label inconsistency }}\}
$$

where $D_{p}\left(f_{p}\right)$ is the data cost of assigning label $f_{p}$ to pixel $p$ and $V\left(f_{p}, f_{q}\right)$ is the label inconsistency cost. $\mathcal{N}$ is a neighborhood system: $(p, q) \in \mathcal{N}$ means $p$ and $q$ are neighbors. Finally, $\lambda$ is a regularization parameter that balances the two costs. Clearly, the role of the label inconsistency cost $V$ is to enhance the robustness of the labeling when the data cost $D_{p}$ is insufficient.

The optimization in Equation (1) is known to be NP-hard [3], except when special assumptions and constraints are applied $[9,4,10,7,1,8]$. Of particular interest is the work by Felzenszwalb and Veksler [1] who describe an $O\left(N^{1.5}\right)$ algorithm for the globally optimal labeling of a three-tiered structure. In that three-tiered structure, each tier is further allowed to be vertically decomposed into an arbitrary number of segments. Here and throughout this paper $N$ refers to the number of image pixels. Unfortunately their algorithm would be too slow for labeling scenes of more than three tiers because of its exponential dependency 

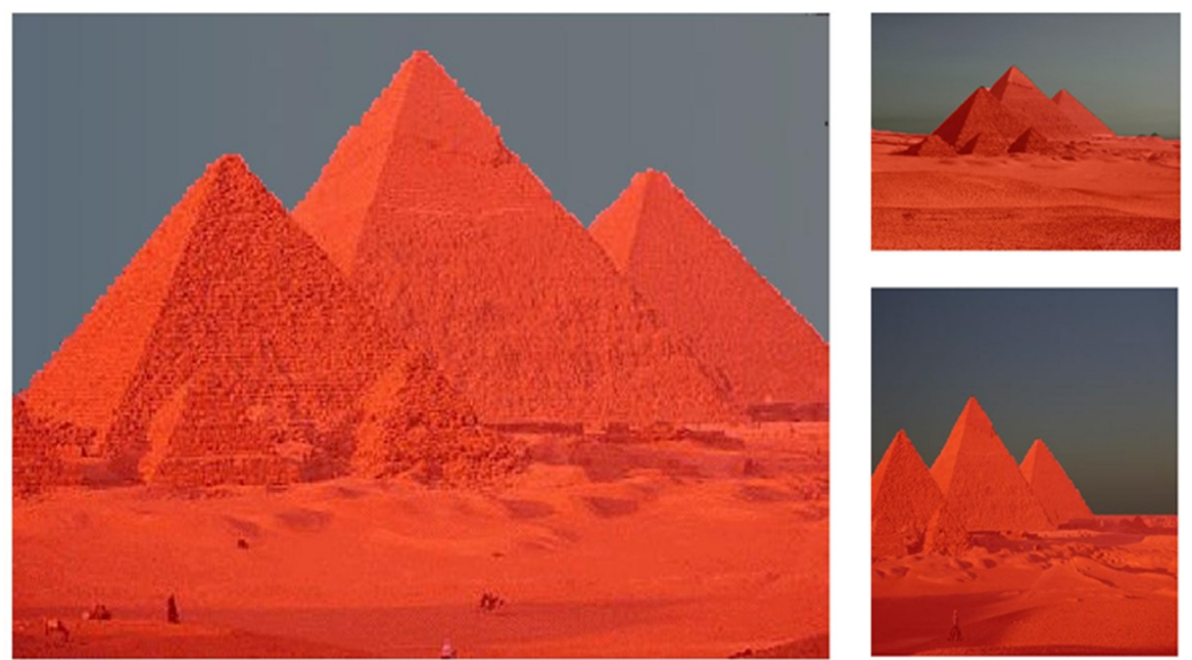

Fig. 2. Although these three pictures differ in scale and perspective, the boundary that separates the sky from the rest has three distinguishable peaks in each image. In this example, the number of local extrema of a boundary curve is a reliable prior that conveys useful domain knowledge.

on the number of tiers. Most recently Strekalovskiy and Cremers [8] extend the computation to multi-tiered labeling using a relaxation of the integer convex program, but with an even greater time complexity. Their algorithm is also approximate due to randomized rounding.

Total variation is the conventional measure of smoothness used to regularize the shape of a tier boundary. However, this measure only encourages a tier boundary to be smooth locally rather than globally. We think that the number of extrema of a boundary curve is a useful topological measure of the degree of global smoothness of a curve. Moreover, the number of extrema of a boundary curve can be used as a prior in tiered labeling and in scene label transfer [11]. This topological measure has been studied in the context of topological persistence and simplification of a triangulated surface $[12,13]$ and recently as a soft prior for one dimensional signal de-noising [14]. To the best of our knowledge this measure has rarely been considered in MRF optimization or in scene label transfer.

\subsection{Our Contributions}

First, under a restricted cost model, we develop an $O(K N)$ approximation algorithm to solve the $K$-tier labeling problem. We find that our algorithm works well in practice and runs in over 100 frames per second on images of resolution $640 \times 480$ when $K$ is less than 6 . When $K=3$, our solution overlaps with the globally optimal one [1] in over $99 \%$ of the pixels but runs 1000 times faster. Second, we propose to use the number of extrema to regularize the smoothness 
of a boundary curve and show that this improves the accuracy of tiered labeling, particularly for indoor scene labeling where the number of local extrema of each boundary curve is known a priori (e.g. the ceiling-wall boundary has one local maximum and the wall-floor boundary has one local minimum). We give an efficient $O(M N)$ algorithm to find an optimal tier boundary with exactly $M$ local extrema using dynamic programming, and demonstrate improved labeling results in detail on a benchmark data set.

\subsection{Organization}

Section 2 presents the general framework of tiered labeling, our chosen restricted cost model, and our linear time approximation algorithm for multi-tiered labeling. Section 3 defines the concept of topological smoothness and presents a linear time algorithm to compute a binary labeling with the boundary curve containing a given number of local maxima and minima. Section 4 tests our algorithm on a selected indoor image data set and compares it to the baseline algorithm of [1] in terms of quality and speed. We show that without sacrificing the quality of the tiered-labeling, our algorithm yields running time improvement of several orders of magnitude. Section 5 concludes.

\section{Tiered Labeling}

The three tiered labeling framework was first studied by Felzenszwalb and Veksler [1]. At a high level, it divides an image into regions of top, middle, and bottom. The middle region is further decomposed into a series of vertical stripes, each with a unique label. We generalize this definition to include multiple tiers. A formal definition is this: Let $\Omega=[1, \cdots, R] \times[1, \cdots, C]$ be an image grid of $R$ rows and $C$ columns. Given a Directed Acyclic Graph (DAG) $\langle\mathcal{L}, \prec\rangle$ where $\mathcal{L}$ is a set of labels and $\prec$ is a partial ordering relation defined in $\mathcal{L}$, we have:

Definition 1 (Tiered Labeling) A labeling function $f: \Omega \rightarrow \mathcal{L}$ is a tiered labeling with respect to $\prec$ if either $f(r, c)=f(r+1, c)$ or $f(r, c) \prec f(r+1, c)$ for each column $1 \leq c \leq C$ and each row $1 \leq r \leq R-1$.

The relation graph can be further decomposed to a set of tiers if we run Breadth-first Search (BFS) on the DAG and group labels that have the same depth from the root into tiers. Note that labels within each tier have no particular ordering between them. In this section we give an approximate labeling algorithm for the $K$-tiered labeling problem. We first show that $1 \mathrm{D}$ tiered labeling can be solved optimally in linear time with respect to the array size, multiplied by the size of relation graph, using dynamic programming. We then solve the $2 \mathrm{D}$ tiered labeling using 1D tiered labeling as submodules for cost approximation. 

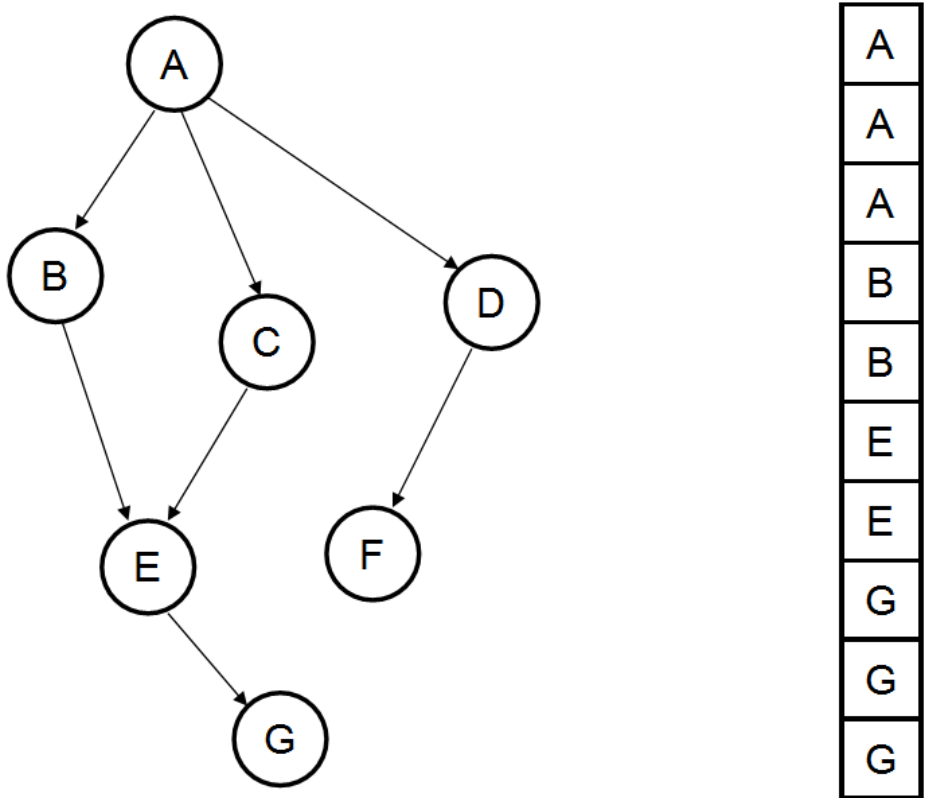

Fig. 3. Left: the "above" relation $\prec$ organized in a directed acyclic graph. Right: one possible tiered labeling in a one dimensional array.

\section{$2.1 \quad$ 1D Tiered Labeling}

We first show that 1D tiered labeling can be optimally solved in $O(E N)$ time on a one dimensional array of size $N$ where $E$ is the number of edges in the relation DAG $\langle\mathcal{L}, \prec\rangle$. The problem is to assign each pixel $1 \leq i \leq N$ a label $f_{i}$ so that either $f_{i}=f_{i+1}$ or $f_{i} \prec f_{i+1}$ for $1 \leq i \leq N-1$. The relation $\prec$ can be understood as "above" and is imposed a priori. Figure 3 illustrates the setting.

We show how to solve the global optimization of Equation (1) using dynamic programming. Let $F(i, l)$ be the optimal cost when position $i$ is labeled $l$. Without loss of generality, $l$ takes positive integer values. We then have the following recursive state equation:

$$
F(i, l)=\min _{l^{\prime}: l^{\prime} \prec l}\{\underbrace{F\left(i-1, l^{\prime}\right)}_{\text {recursion }}+\underbrace{V\left(l^{\prime}, l\right)}_{\text {label inconsistency }}+\underbrace{D_{i}(l)}_{\text {data cost }}\}
$$

Dynamic programming computes $F(i, l)$ for each $1 \leq i \leq N$ and each $1 \leq$ $l \leq K$. Since each edge is visited once at each $i$, the overall time complexity is $O(E N)$. For the boundary conditions we specify: $F(1, l)=D_{1}(l)$ for each $l \in \mathcal{L}$.

We point out that for $1 \mathrm{D}$ tiered labeling, both the data cost $D$ and the pairwise potential $V$ are allowed to take arbitrary forms. 


\subsection{D Tiered Labeling}

While the 1D tiered labeling problem can be optimally solved efficiently for arbitrary number of tiers, the 2D tiered labeling is far more difficult. In fact, it is NP-hard to compute the general 2D tiered labeling problem as it is as difficult as solving the general 2D MRF. We look for approximation algorithms instead.

Three simplifications are made for efficient computation. First, as a preprocessing step we aggregate the cost of multiple labels within a single tier into a single cost function. Let $D^{k}$ be the aggregate cost of tier $k$. The set of object labels in tier $k$ is denoted $\mathcal{L}_{k}$, a subset of $\mathcal{L}$. We define for each pixel $p$ :

$$
D^{k}(p)=\min _{l \in \mathcal{L}_{k}} D_{p}(l) .
$$

Then, the modified relation graph $\mathcal{L}$ is reduced to $K$ tiers, each with a single label. In other words, the modified relation graph has $K$ nodes and $K-1$ edges, organized as a linear chain. We argue that this way of compressing the relation graph does not cause serious problems as after assigning the tiered labels under the modified relation graph, one can unfold the collapsed labels in each tier.

In the second simplification, we divide the $K$-tier labeling to a series of $K-1$ binary labeling problems. Each binary labeling problem can be solved in $O(N)$ time. First, we use the $1 \mathrm{D}$ tiered labeling algorithm to compute the cumulative cost $F_{c}$ for each column $c . F_{c}(i, k)$ is therefore the optimal cost of labeling position $i$ as $k$ at column $c$, and can be computed using Equation (2). Since the modified relation graph is a linear chain, we label each tier as $1,2, \cdots, K$ from top to bottom. We start from the bottom tier and separate it from the rest of the tiers.

In the third simplification, we restrict the pairwise potential $V$. Specifically, $V\left(f_{p}, f_{q}\right)$ can be arbitrary for $(p, q) \in \mathcal{N}$ in the same column. However, when $(p, q) \in \mathcal{N}$ are in the same row, we take:

$$
V\left(f_{p}, f_{q}\right)=\left\{\begin{array}{l}
1 \text { if } f_{p} \neq f_{q} \\
0 \text { otherwise }
\end{array} .\right.
$$

In other words, the pairwise potential is allowed to take an arbitrary form along columns and takes the form of a Potts model along rows. Combining the second and the third simplifications, the binary labeling problem is equivalent to finding a single path $\left\{x_{c}\right\}_{c=1}^{C}$ of row indices for each column. This path separates the bottom tier from the one above it. The problem formulation is therefore:

$$
\min _{x_{1}, \cdots, x_{C}}\{\sum_{c=1}^{C} \mu_{c}\left(x_{c}\right)+\lambda \underbrace{\sum_{c=1}^{C-1}\left|x_{c+1}-x_{c}\right|}_{\text {label inconsistency }}\}
$$

where

$$
\mu_{c}\left(x_{c}\right) \triangleq F_{c}\left(x_{c}, k-1\right)+\sum_{r=x_{c}+1}^{R} D^{k}(r, c)
$$



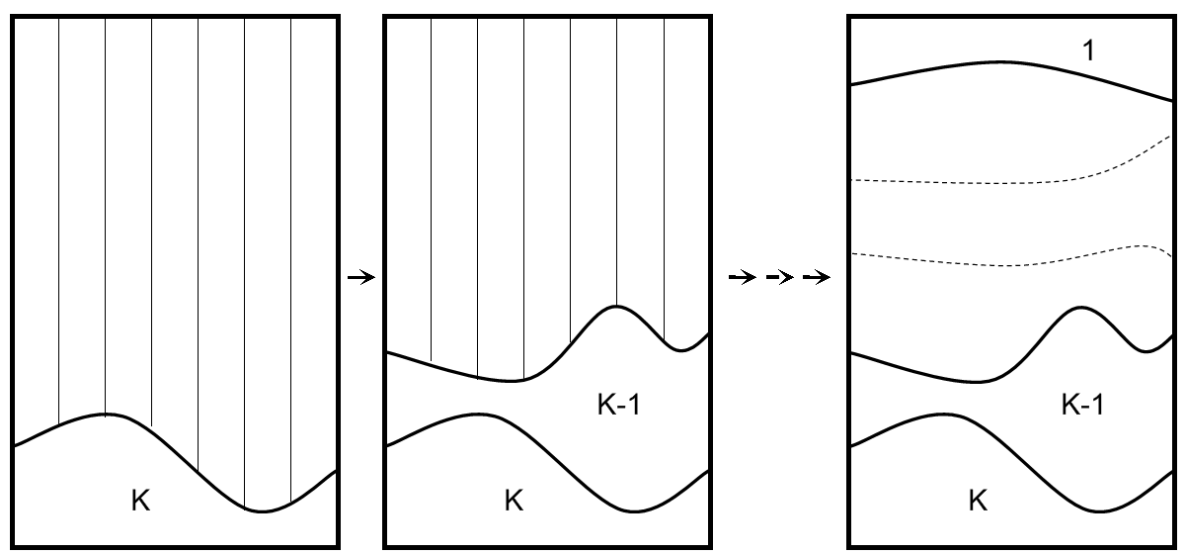

Fig. 4. Decomposing a $K$-tiered labeling to a series of $K-1$ binary labeling.

stands for the data cost and can be evaluated in $O(1)$ time if an integral image is pre-computed for $D^{k}$. Let $E_{c}\left(x_{c}\right)$ be the optimal cost up to column $c$ at pixel $x_{c}$. The recursive state equation for the global minimization is:

$$
E_{c}\left(x_{c}\right)=\mu_{c}\left(x_{c}\right)+\min _{1 \leq x_{c-1} \leq R}\left\{E_{c-1}\left(x_{c-1}\right)+\lambda\left|x_{c}-x_{c-1}\right|\right\}
$$

Thanks to the generalized distance transform [15], Equation (7) can be evaluated in $O(R)$ time. Since this dynamic program takes $C$ steps, the overall time complexity is $O(R C)$ or $O(N)$. Once tier $k$ is separated, we proceed to tier $k-1$ and separate it from the tiers above it in a similar way. Since each time the binary labeling takes $O(N)$ time, the overall time complexity is $O(K N)$. Figure 4 illustrates this greedy construction.

The first simplification compresses the labeling graph into a linear chain. Once the tiered labeling is done, one can further decompose each tier into vertical bands to uncover possible multiple labels (Figure 5). This is essentially a one dimensional problem because each column within each tier can only have one label. Consider tier $t$ and its label set $\mathcal{L}_{t}$. Let $C(i, l)$ be the optimal cost of labeling column $i$ as label $l$. Let $D(i, l)$ be the data cost of labeling column $i$ as $l$. The state equation for recovering the labels within tier $t$ is:

$$
C(i, l)=\min \left\{\lambda+\min _{l^{\prime} \in \mathcal{L}_{t}, l^{\prime} \neq l} C\left(i-1, l^{\prime}\right), C(i-1, l)\right\}+D(i, l) .
$$

The time complexity for the dynamic program above is linear with respect to the number of columns, multiplied by the square of the number of labels within a tier. Since the number of labels is typically much smaller than the number of rows of an image, the complexity can be safely neglected compared to the main $O(K N)$ algorithm. 

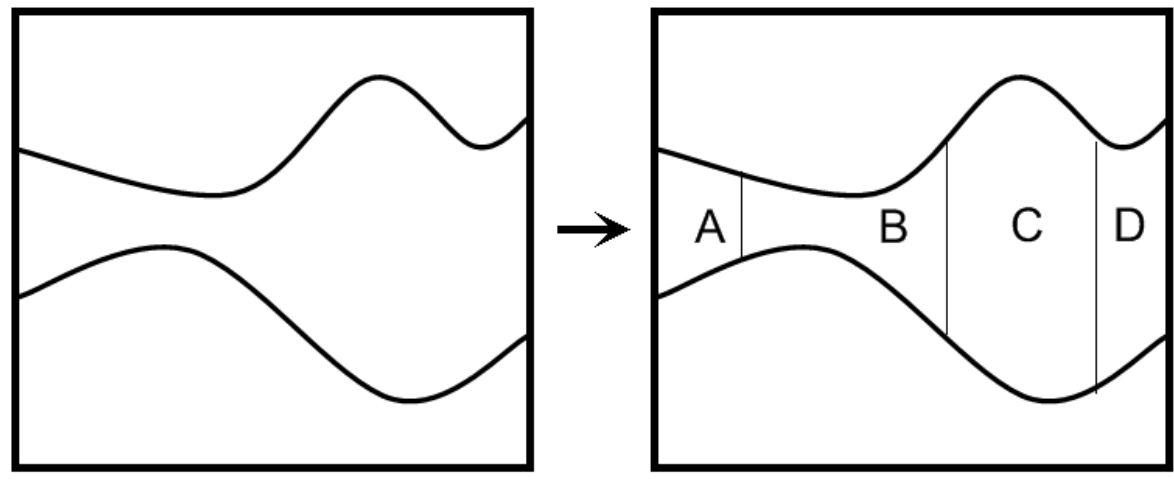

Fig. 5. Unfolding object labels by vertical decomposition.

Because of the three simplifications made, our algorithm does not minimize the exact MRF energy function in Equation (1). However, our algorithm guarantees that the solution is a tiered labeling by construction. The advantages of our algorithm lie in its practical efficiency of $O(N K)$ complexity, the ability to label multiple tiers beyond three, and good performance on par with other methods of greater complexity. For instance, although the algorithm given in [1] is globally optimal when $K=3$, our solution differs from the globally optimal one in less than $1 \%$ of the total pixels and runs over 1000 times faster than the $O\left(N^{1.5}\right)$ algorithm in [1] in our experiments.

\section{Topological Smoothness}

In the discussion above we use the label inconsistency cost of Equation (4). While this penalty function works generally well in practice, it induces a large penalty for sharp transitions (Figure 6). Moreover, the total variation only quantifies the local smoothness of a curve. Many scenes have tier boundaries that are globally smooth in the sense that the borders contain only one or two local extrema. For instance, in the work of [7], a scene is decomposed into top, left, right, bottom and middle and the top and bottom tier boundaries have only one local minimum and one local maximum respectively due to their polygonal representation.

We propose to use the number of extrema of a path to quantify its topological smoothness. Our algorithm finds a minimal cost path with exactly $M$ local extrema, which is useful for the binary labeling problem described in the previous section. Note that the new prior cannot be modeled appropriately in an MRF formulation. Our algorithm can also be modified to find a path with at most $M$ local extrema or $M$ local maxima, all with the same asymptotic complexity. Let $F_{c}\left(x_{c}\right)$ be the total data cost of column $c$ if the path passes through $x_{c}$. Here one 
can simply evaluate $F_{c}\left(x_{c}\right)$ in $O(1)$ time using a pre-computed integral image representation. The objective under the topological smoothness prior is:

$$
\min _{x_{1}, \cdots, x_{C}} \sum_{c=1}^{C} F_{c}\left(x_{c}\right)
$$

subject to: path $\left\{x_{c}\right\}_{c=1}^{C}$ has $M$ local extrema

In this constrained optimization, we omit the label inconsistency cost because we expect to use the number of extrema of the path to automatically enhance its regularity. However, including the pairwise smoothness term: $\lambda \sum_{c=1}^{C-1}\left|x_{c+1}-x_{c}\right|$ does not increase the computational complexity of our algorithm thanks again to the generalized distance transform. For ease of description we omit this term in the rest of the discussion. The notion of a local maximum or minimum is this:

Definition 2 (Local Extrema) An interval $[I, J]$ is said to be a local maximum of $\left\{x_{c}\right\}_{c=1}^{C}$ if $x_{I-1}<x_{I}=x_{I+1}=\cdots=x_{J}>x_{J+1}$. The interval $[I, J]$ is said to be a local minimum of $\left\{x_{c}\right\}_{c=1}^{C}$ if it is a local maximum of $\left\{-x_{c}\right\}_{c=1}^{C}$.

Let $C(r, c, m, \uparrow)$ be the optimal cumulative cost of the path that contains $m$ local extrema before reaching pixel $(r, c)$ through an ascending direction. Similarly, let $C(r, c, m, \downarrow)$ be the optimal cumulative cost of the path that contains $m$ local extrema before reaching pixel $(r, c)$ though a descending direction. We have the following alternating state equations for dynamic programming:

$$
\begin{array}{r}
C(r, c, m, \uparrow)=F_{c}(r)+\min \left\{\min _{r^{\prime} \geq r} C\left(r^{\prime}, c-1, m, \uparrow\right),\right. \\
\left.\min _{r^{\prime}>r} C\left(r^{\prime}, c-1, m-1, \downarrow\right)\right\} \\
C(r, c, m, \downarrow)=F_{c}(r)+\min _{\min ^{\prime} \leq r} C\left(r^{\prime}, c-1, m, \downarrow\right), \\
\left.\min _{r^{\prime}<r} C\left(r^{\prime}, c-1, m-1, \uparrow\right)\right\}
\end{array}
$$

These state equations utilize the fact that a local maximum is created by an ascending path followed by a descending path and a local minimum is created by a descending path followed by an ascending path. The use of relation symbols $\geq$ and $\leq$ include the equality relations so that the path is allowed to move in the same row across adjacent columns.

The boundary conditions need to be posed carefully. First, when $c=0$, we have for each $1 \leq r \leq R$ and $1 \leq m \leq M$ the following boundary condition:

$$
C(r, 0, m, \uparrow)=C(r, 0, m, \downarrow)=+\infty
$$

Second, when $k=0$, we pre-compute the data cost:

$$
B(r, c)=\left\{\begin{array}{rl}
F_{c}(r) & \text { if } c=0 \\
B(r, c-1)+F_{c}(r) & \text { otherwise }
\end{array} .\right.
$$




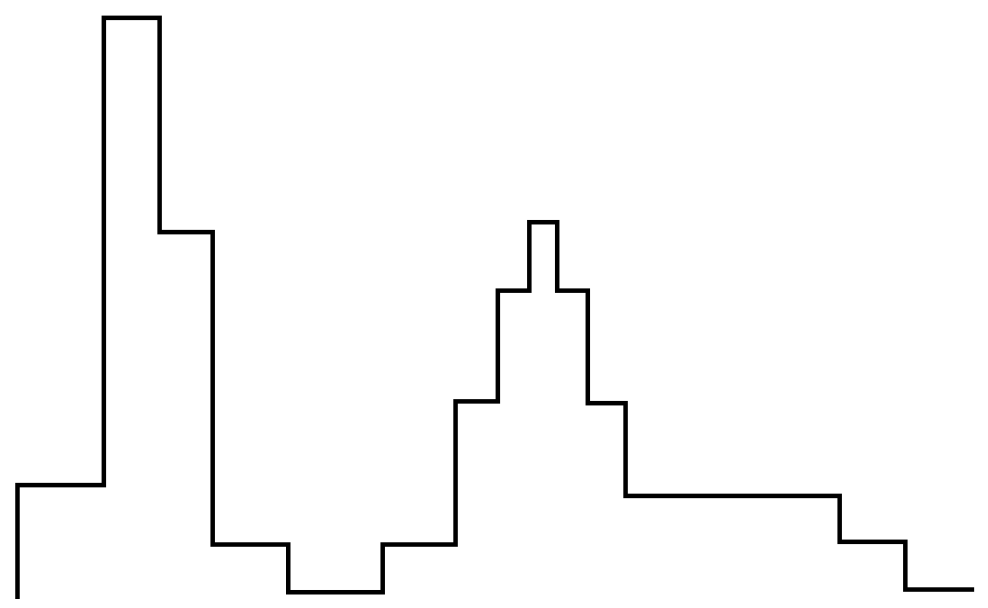

Fig. 6. A cartoon city skyline. This tier boundary induces a large label inconsistency by Equation (4) due to sharp transitions. However, the cost is low by the standard of topological smoothness as the skyline contains only two local maxima. The number of local maxima is a topological quantity that tolerates significant sharp transitions.

The boundary conditions for $C$ are:

$$
\begin{aligned}
& C(r, c, 0, \uparrow)=F_{c}(r)+\min \left\{\min _{r^{\prime} \geq r} C\left(r^{\prime}, c-1,0, \uparrow\right), \min _{r^{\prime}>r} B(r, c-1)\right\} \\
& C(r, c, 0, \downarrow)=F_{c}(r)+\min \left\{\min _{r^{\prime} \leq r} C\left(r^{\prime}, c-1,0, \downarrow\right), \min _{r^{\prime}<r} B(r, c-1)\right\}
\end{aligned}
$$

The boundary conditions rule out the case that a constant level curve followed by an ascending or descending path may accidentally induce a local maximum or minimum. The correctness of the dynamic programming then follows easily by induction. The overall time complexity is $O(M N)$ by following the state equation and using a simple book-keeping method for updating the running min in a sequential way.

\section{Experiments}

We demonstrate applications of multi-tier labeling with and without the topological smoothness constraint. We compare our approximation algorithm to the $O\left(N^{1.5}\right)$ algorithm by Felzenszwalb and Veksler [1] on labeling a three-tiered structure. Although their algorithm is globally optimal in terms of solving Equation (1), we show that our greedy construction achieves similar results but runs much faster in practice. We collect 60 images from two benchmark data sets [16, 17] and annotate the ground truth of a three tiered labeling. For fair comparison, both algorithms use the same feature costs. All the data set, the annotation, our $\mathrm{C}++/$ MATLAB implementation, the generated costs, and test files 
Table 1. Average accuracy over 60 images. "+Topology" refers to our tiered labeling under the topological smoothness constraint: the top tier boundary has one local minimum and the bottom tier boundary has one local maximum. Global accuracy is calculated on the whole image. Detail accuracy is counted on the cropped image. See also Figure 8 for detailed visual comparison.

\begin{tabular}{lllll}
\hline & naive & DP [1] & Ours & +Topology \\
\hline Global Accuracy & 0.80 & 0.96 & 0.96 & $\mathbf{0 . 9 7}$ \\
Detail Accuracy & 0.76 & 0.90 & 0.91 & $\mathbf{0 . 9 4}$ \\
Timing (seconds) & - & 10.1 & $<0.01$ & $<0.01$ \\
Overlap with [1] & - & 1 & $>0.99$ & - \\
\hline
\end{tabular}

are available in the supplementary file and will be available at authors' website: http://www.cs/duke/edu/ yuanqi.

We generate the pixel costs as follows: Let $T, O, B$ be the collection of sampled $3 \mathrm{D}$ color vectors associated to the top, middle and bottom tier. Let $d(p)$ be the color vector at pixel $p$. We compute the ratio:

$$
\gamma(p)=\frac{\min _{d \in T}\|d(p)-d\|}{\min _{d \in O \cup B}\|d(p)-d\|}
$$

and we assign the cost associated to $T$ based on the ratio:

$$
f_{T}(p)= \begin{cases}+1 & \text { if } \gamma(p)>\frac{3}{2} \\ -1 & \text { if } \gamma(p)<\frac{2}{3} \\ +\frac{1}{2} & \text { otherwise }\end{cases}
$$

The rationale behind the cost design is that the closer the feature resembles $T$ 's features relative to the background features of $O$ and $B$, the lower the cost. Ambiguous features would receive a cost that is positive. $f_{O}(p)$ and $f_{B}(p)$ are generated similarly under cyclic permutation of $T, O, B$. Since each tier is composed of a single object, there is no need to invoke the step for recovering multiple objects within a tier.

Figure 7 displays sample results and Table 1 shows numerical comparisons. In summary, our algorithm runs 1000 times faster than [1] on test images and differs from the optimal solution in less than $1 \%$ of all the pixels. Accuracy is further improved under the topological prior. This improvement is not obvious if tested on the whole image due to the fact that boundaries are thin. To magnify this advantage we crop the boundary regions and test accuracy on this smaller area (Figure 8). We achieve 4\% improvement over Felzenszwalb and Veksler [1] in details. Figure 9 shows that the topological prior tolerates sharp transitions while traditional smoothness priors may fail. 

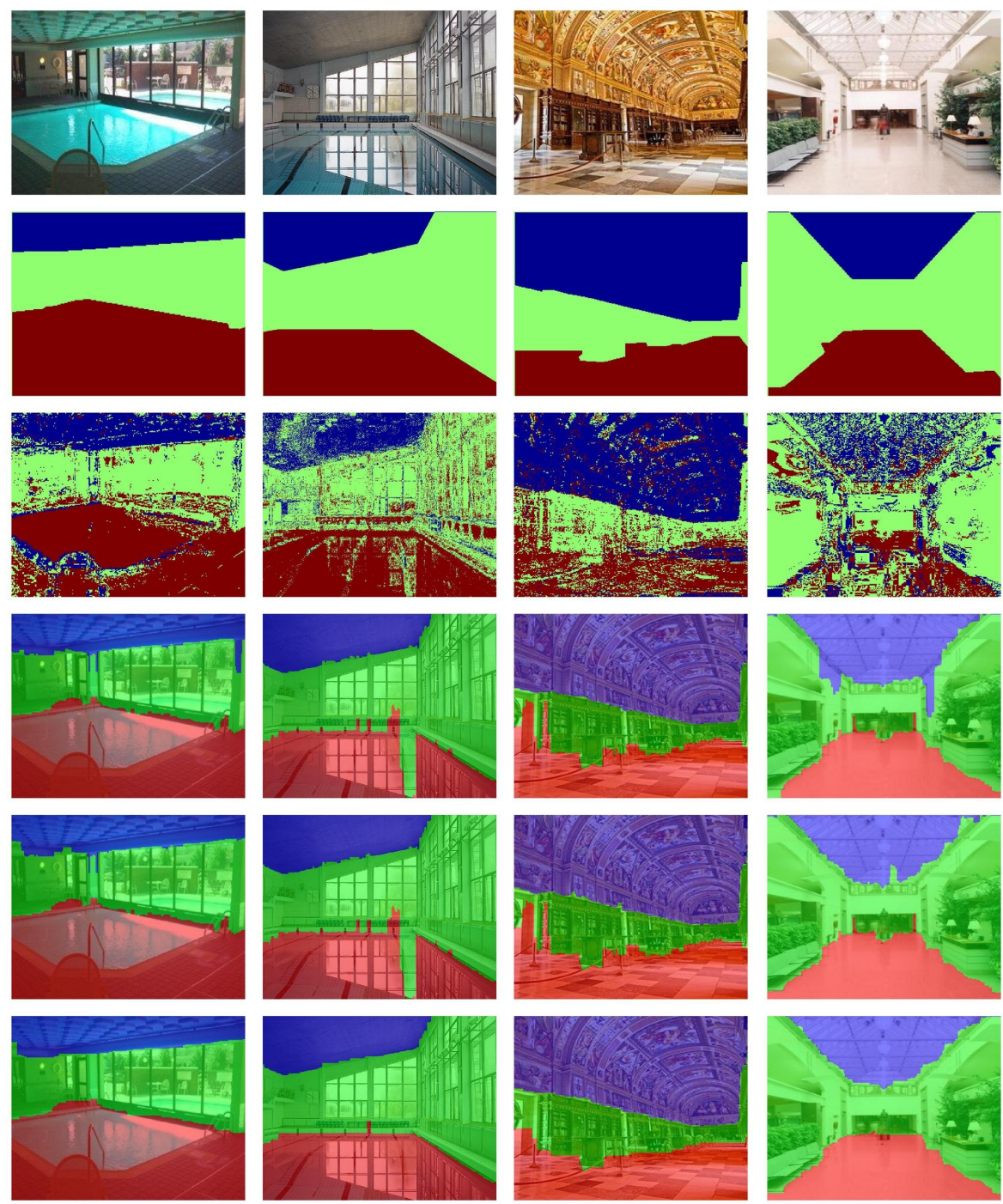

Fig. 7. Each row shows 4 out of 60 test images. From top to bottom: Input image, ground truth, generated cost, tiered labeling of [1], our result, our result under topological smoothness constraint. Best viewed when enlarged and in color.

\section{Conclusions}

We present an $O(K N)$ greedy algorithm for the $K$-tier labeling of an image of $N$ pixels. In addition, we propose to use a novel topological prior to regularize 

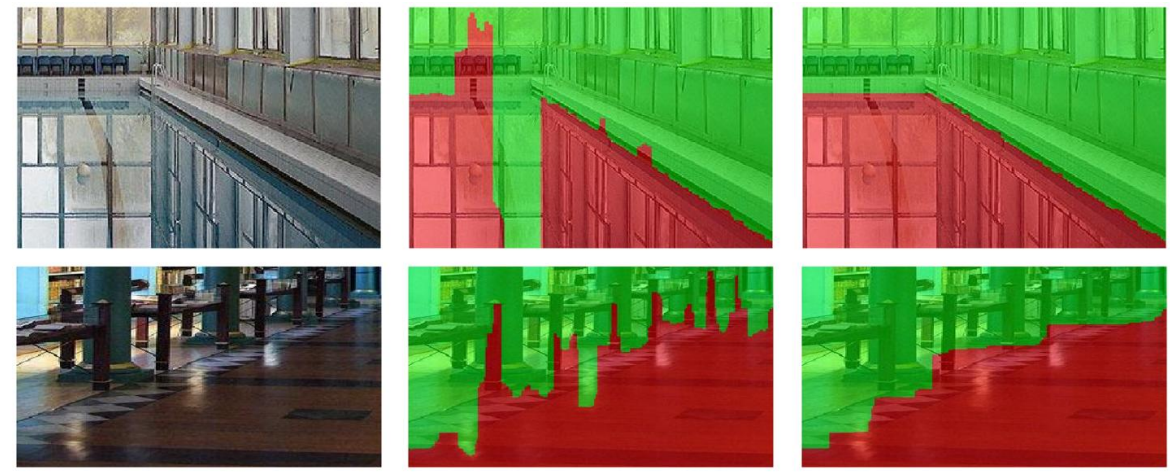

Fig. 8. Left to right: the cropped image, result by [1], and our labeling with topological constraints (1 local extremum allowed).

the tier boundaries and present an $O(M N)$ algorithm for finding a minimalcost binary labeling with exactly $M$ local extrema on the border. Our algorithm for multi-tier scene labeling runs much faster than the previous method without sacrificing the labeling accuracy. The accuracy is further improved under the topological prior, which is simple in concept and equally efficient in implementation. One interesting question is whether our algorithm has a non-trivial theoretical approximation bound relative to the globally optimal solution.

Acknowledgement: This work is supported by the Army Research Office under Grant No. W911NF-10-1-0387 and by the National Science Foundation under Grant $I I S-10-17017$.

\section{References}

1. Felzenszwalb, P., Veksler, O.: Tiered scene labeling with dynamic programming. In: IEEE CVPR. (2010) 3097-3104

2. Li, S.: Markov random field modeling in computer vision. Computer science workbench. Springer (1995)

3. Boykov, Y., Veksler, O., Zabih, R.: Fast approximate energy minimization via graph cuts. IEEE PAMI 23 (2001) 1222-1239

4. Ishikawa, H.: Exact optimization for markov random fields with convex priors. IEEE PAMI 25 (2003) 1333-1336

5. Winn, J., Shotton, J.: The layout consistent random field for recognizing and segmenting partially occluded objects. In: IEEE CVPR. (2006) 37-44

6. Kohli, P., Ladicky, L., Torr, P.: Robust higher order potentials for enforcing label consistency. IJCV 82 (2009) 302-324

7. Liu, X., Veksler, O., Samarabandu, J.: Order-preserving moves for graph-cut-based optimization. IEEE PAMI 32 (2010) 1182-1196

8. Strekalovskiy, E., Cremers, D.: Generalized ordering constraints for multilabel optimization. In: ICCV. (2011) 

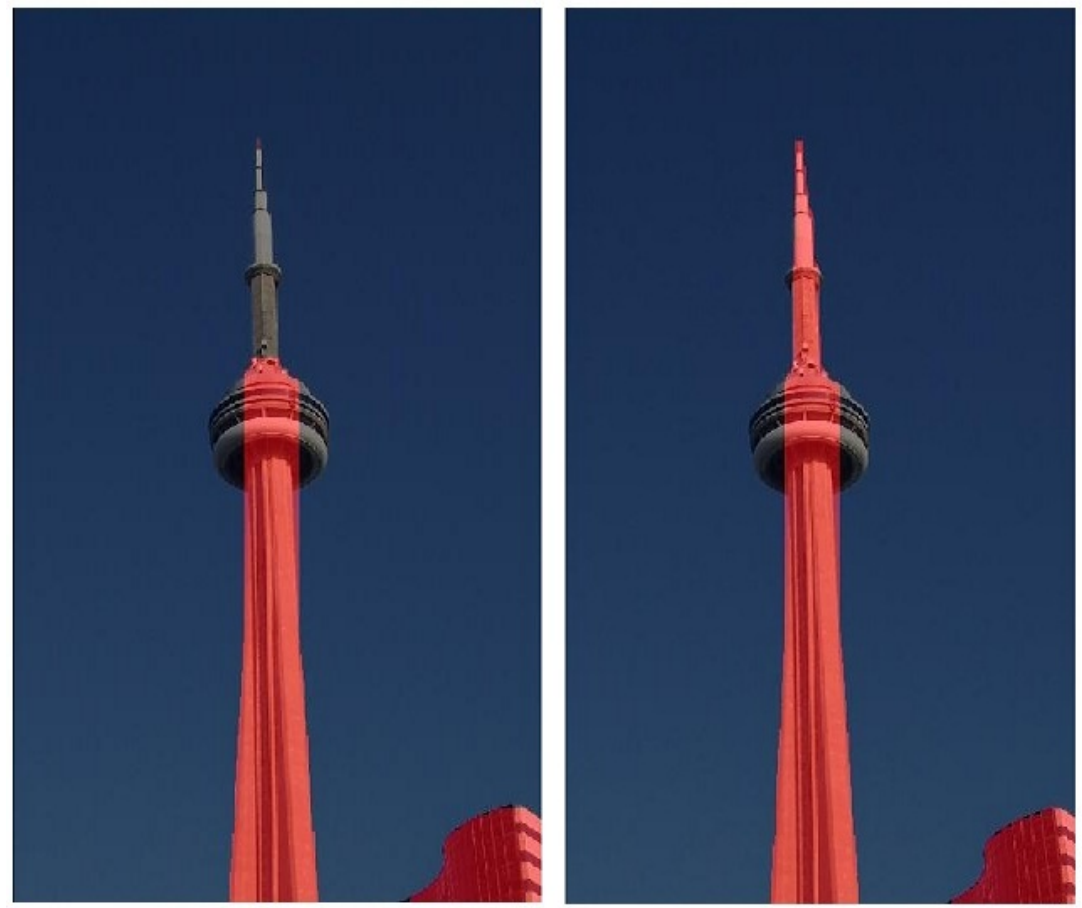

Fig. 9. Traditional prior such as total variation misses the sharp transition (left). Our topological prior respects sharp transitions (right).

9. Greig, D., Porteous, B., Seheult, A.: Exact maximum a posteriori estimation for binary images. Journal of the Royal Statistical Society 51(2) (1989) $271-279$

10. Kolmogorov, V., Rother, C.: Minimizing nonsubmodular functions with graph cuts-a review. IEEE PAMI 29 (2007) 1274-1279

11. Liu, C., Yuen, J., Torralba, A.: Sift flow: Dense correspondence across scenes and its applications. IEEE PAMI 33 (2011) 978-994

12. Edelsbrunner, H., Letscher, D., Zomorodian, A.: Topological persistence and simplification. Discrete \& Computational Geometry 28 (2002) 511-533

13. Edelsbrunner, H., Harer, J., Zomorodian, A.: Hierarchical morse-smale complexes for piecewise linear 2-manifolds. Discrete \& Computational Geometry 30 (2003) $87-107$

14. Gu, S., Zheng, Y., Tomasi, C.: Oscillation regularization. In: The 37th International Conference on Acoustics, Speech, and Signal Processing. (2012)

15. Felzenszwalb, P., Huttenlocher, D.: Distance transforms of sampled functions. Technical Report TR2004-1963, Cornell Computing and Information Science (2004)

16. Oliva, A., Torralba, A.: Modeling the shape of the scene: A holistic representation of the spatial envelope. IJCV 42 (2001) 145-175

17. Quattoni, A., Torralba, A.: Recognizing indoor scenes. In: IEEE CVPR. (2009) 413-420 